\title{
On Graphs whose Spread is Maximal
}

\author{
T. Aleksić, M. Petrović
}

\begin{abstract}
A graph's spread is defined as the difference between the largest eigenvalue and the least eigenvalue of the graph's adjacency matrix. Characterizing a graph with maximal spread is still a difficult problem. If we restrict the discussion to some classes of connected graphs of a prescribed order and size, it simplifies the problem and it may allow us to solve it. Here, we discuss some results on graphs whose spread is maximal in certain classes of graphs.
\end{abstract}

Keywords: Graph, cactus, spread, least eigenvalue, index

\section{Introduction}

Let $G$ be a simple graph with vertex set $V(G)$ and edge set $E(G)$. The graph $G$ is of order $n=|V(G)|$ and size $m=|E(G)|$. Let $A(G)$ be the $(0,1)$-adjacency matrix of $G$. Since $A(G)$ is symmetric, its eigenvalues are real. Without loss of generality we can order them nonincreasingly as $\lambda_{1}(G) \geq \lambda_{2}(G) \geq \cdots \geq \lambda_{n}(G)$ and refer to them as the eigenvalues of $G$. The largest eigenvalue of $G$ is denoted by $\rho(G)$ and called the spectral radius or index and the least eigenvalue of $G$ is denoted by $\lambda(G)$. A graph's spread $s(G)$ is defined as the difference between the index and the least eigenvalue of the graph, i. e. $s(G)=\rho(G)-\lambda(G)$.

Interest in studying the spread of graphs belonging to some prescribed classes has increased in the last several years. In 1983 Petrovi [5] determined all connected graphs whose spread does not exceed 4. In 2001 Gregory et al. [4] presented some lower and upper bounds for the spread of a graph. They showed that a path is the unique graph with minimal spread among the connected graphs of a given order. The star and the path have the maximal spread and the minimal spread among all trees with given order, respectively. Gregory et al. [4] also give the following conjecture.

Conjecture 1 Among all graphs with $n$ vertices and $m<\left\lfloor\frac{n^{2}}{4}\right\rfloor$ edges, if $G$ is one with maximal spread, then $G$ must be bipartite.

Manuscript received November 21, 2014; accepted March 4, 2015.

T. Aleksić is with the Faculty of Science, University of Kragujevac, Kragujevac, Serbia, M. Petrović is with the State University of Novi Pazar, Novi Pazar, Serbia; 


\section{Unicyclic graphs}

In 2008 Fan, Wang and Gao [3] characterized a unique graph whose spread is maximal among all unicyclic graphs of order $n$. A graph is unicyclic if it has $n$ vertics and $m=n$ edges.
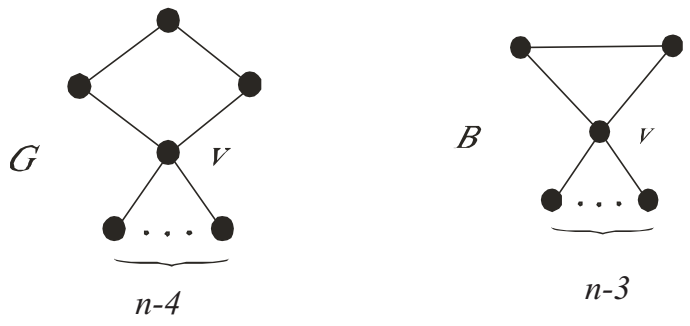

Fig. 1. The graphs $U_{1}$ i $U_{2}$

Theorem 1 ([3]) The graph $U_{1}$ (Fig. 1) is a unique graph with maximal spread in the class of connected unicyclic graphs of order $n$ for $4 \leq n \leq 5$, and the graph $U_{2}$ (Fig. 1) a unique graph with maximal spread in the same class for $n \geq 6$.

It is easy to see that this result shows that Conjecture 1 is not valid in general. Graphs with maximal spread were also studied in the class of cacti.

\section{Cacti}

A connected graph $G$ is a cactus if any two of its cycles have at most one common vertex. If all cycles of the cactus $G$ have exactly one common vertex, say $v_{0}$, then it is called a bundle. The vertex $v_{0}$ is called the central vertex of a bundle.

Observing the class of cacti with $n$ vertices and $k$ cycles, in 2013 we [1] determined a unique graph with maximal spread in this class. Furthermore, we proved that the graph with maximal spread among cacti with $n$ vertices and $k$ cycles is a bundle.

In what follows we use the following notation: $C(n, k)$ denotes the set of all cacti with $n$ vertices and $k$ cycles and $B(n, k)$ denotes the set of all bundles with $n$ vertices and $k$ cycles. The graph depicted in Figure 2 is denoted by $G\left(C_{1}, \ldots, C_{k} ; r\right)(k \geq 1, r \geq 0)$ and $S(n, k)$ is the set of bundles in $B(n, k)$ of the form $G\left(C_{1}, \ldots, C_{k} ; r\right)$.

Let $B^{*}(n, k)$ be the set of bundles in $S(n, k)$ for which the length of the longest cycle is less than or equal to 4 . Also, let $t$ be the maximal number of cycles of length 4 . Obviously, $t=\min \{k, n-(2 k+1)\}$. Denote by $B_{k, s}=B(k-s, s, n-(2 k+s+1))(s=0,1, \ldots, t)$ the graph in $B^{*}(n, k)$ which contains $k-s$ cycles of length $3, s$ cycles of length 4 and $n-(2 k+s+1)$ pendant vertices attached to $v_{0}$ (Figure 3). Then $B^{*}(n, k)=\left\{B_{k, 0}, \ldots, B_{k, t}\right\}$. 


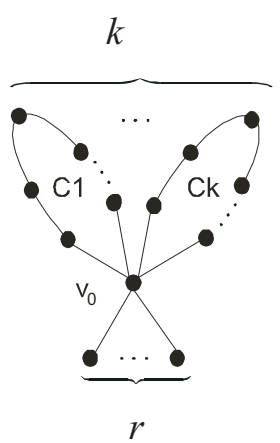

Fig. 2. The bundle $G\left(C_{1}, \ldots, C_{k} ; r\right)$

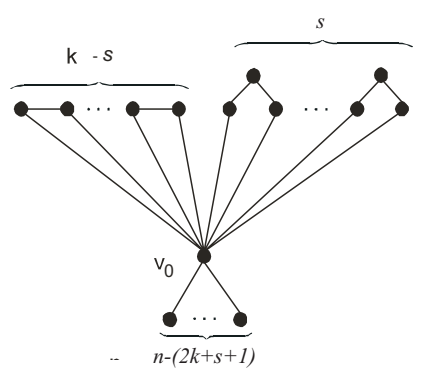

Fig. 3. The bundle $B_{k, s}$

Bearing in mind that a graph's spread is closely related to the graph's index and the least eigenvalue of the graph, the following lemma was very important for proving the main result.

Lemma 1 ([1]) For any graph $G \in C(n, k) \backslash S(n, k)$ there exists a graph $B \in S(n, k)$ such that graphs $G$ and $B$ have the same cycle lengths and the following inequalities are satisfied:

1. $\lambda(B)<\lambda(G)$

2. $\rho(B)>\rho(G)$,

3. $s(B)>s(G)$.

Corollary 1 ( [1]) Let $G$ be a graph with maximal spread in the class $C(n, k) k \geq 1, n \geq$ $2 k+1)$, then $G \in B^{*}(n, k)$.

The main result in the class of cacti with $n$ vertices and $k$ cycles is presented in the next theorem. 
Theorem 2 ( [1]) Let $G$ be a graph with maximal spread in the class $C(n, k) k \geq 1, n \geq$ $2 k+1)$ and let $t=\min \{k, n-(2 k+1)\}$. Then $G \cong B(k-t, t, n-(2 k+t+1))$ if $n \in\{4,5\}$ and $k=1$, or $n=6$ and $k=2$, and $G \cong B(k, 0, n-(2 k+1))$ in all other cases.

\section{Bicyclic graphs}

In 2011 Petrovi, Borovianin and Aleksi [6] determined a unique graph with maximal spread in the set of connected bicyclic graphs of order $n$, when $n>27$. A graph with $n$ vertices and $m=n+1$ edges is called bicyclic.
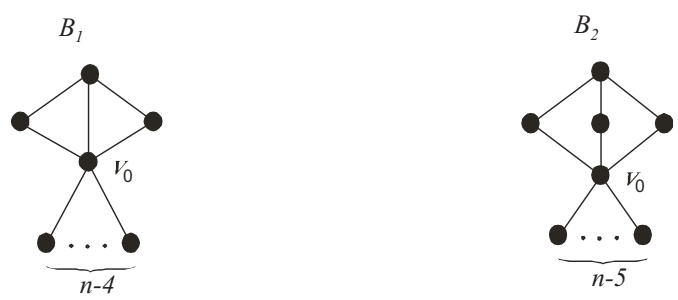

Fig. 4. The graphs $B_{1}$ i $B_{2}$

Theorem 3 ( [6]) The graph $B_{1}$ (Fig. 4) is a unique graph with maximal spread within the class of connected bicyclic graphs of order $n>27$.

Recently, in [2] we determined a unique graph with maximal spread in the class of connected bicyclic graphs of order $n$, for any $n \in N$, using the following idea. If we denote by $\mathscr{B}(n)$ the set of all connected bicyclic graphs with $n$ vertices, then

$$
\mathscr{B}(n)=\mathscr{B}_{1}(n) \bigcup \mathscr{B}_{2}(n),
$$

where $\mathscr{B}_{1}(n)$ and $\mathscr{B}_{2}(n)$ are defined as follows. The set $\mathscr{B}_{1}(n)$ contains graphs from $\mathscr{B}(n)$ in which any two cycles have at most one common vertex. Similarly, $\mathscr{B}_{2}(n)$ is the set of graphs from $\mathscr{B}(n)$ which contain two cycles that have more than one common vertex. Obviously, $\mathscr{B}_{1}(n) \cap \mathscr{B}_{2}(n)=\emptyset$.

Note that the set $\mathscr{B}_{1}(n)$ can be seen as the set of cacti with $n$ vertices and two cycles. Hence, the following result holds.

Lemma 2 ( [1]) Let $G$ be a graph with maximal spread in the class $\mathscr{B}_{1}(n)$. If $n=6$ then $G \cong B_{4}$ and if $n \geq 5$ and $n \neq 6$ then $G \cong B_{3}$ (Fig. 5).

In the set $\mathscr{B}_{2}(n)$ we proved the next lemma. 

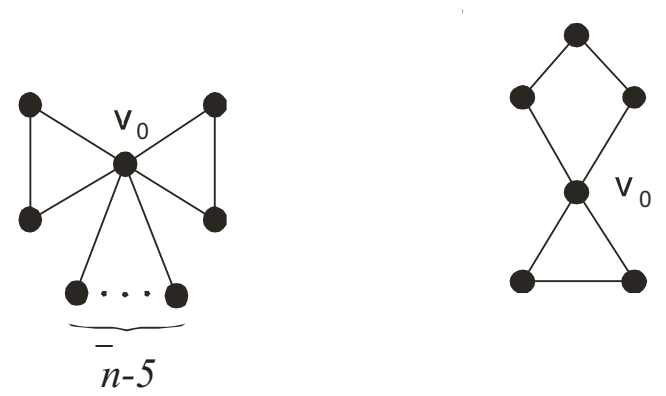

Fig. 5. The graphs $B_{3}$ and $B_{4}$

Lemma 3 ( [2]) Let $G$ be a graph from the set $\mathscr{B}_{2}(n)$.

1. If $5 \leq n \leq 8$, then $s(G) \leq s\left(B_{2}\right)$ and the equality holds if and only if $G \cong B_{2}$ (Fig. 4).

2. If $n=4$ or $n \geq 9$, then $s(G) \leq s\left(B_{1}\right)$ and the equality holds if and only if $G \cong B_{1}$ (Fig. 4).

Combining Lemmas 2 and 3 the main result in this class was proved.

Theorem 4 ( [2]) Let $G$ be a connected bicyclic graph of order $n$.

1. If $5 \leq n \leq 8$, then $s(G) \leq s\left(B_{2}\right)$ and the equality holds if and only if $G \cong B_{2}$ (Fig. 4).

2. If $n=4$ or $n \geq 9$, then $s(G) \leq s\left(B_{1}\right)$ and the equality holds if and only if $G \cong B_{1}$ (Fig. 4).

\section{Conclusion}

Graphs with maximal spread have been investigated in some other classes too. In 2011 Petrovi, Aleksi and Simi [7] identified the graphs with the largest spread within connected graphs of order $n$ and size $n+k(k \in\{0,1,3,4\})$, when $n$ is large enough.

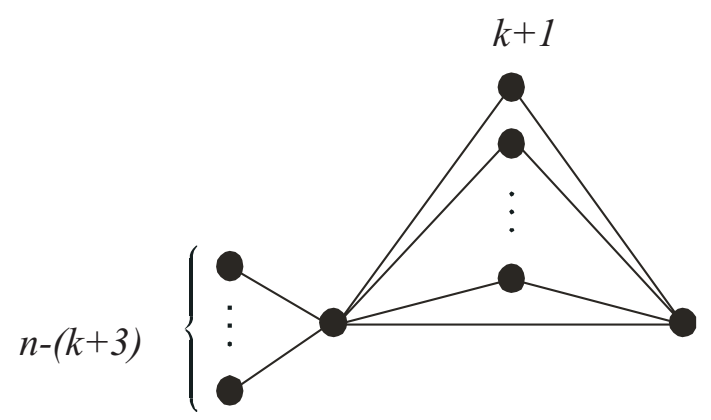

Fig. 6. he graph $G_{0}$ 
Theorem 5 ([7]) Let $G_{0}$ be the graph shown in the Figure 6. Also, let $k \in\{0,1,3,4\}$ be fixed number and let $G$ be a graph of order $n$ and size $n+k$. If $n$ is sufficiently large then

$$
s(G) \leq s\left(G_{0}\right),
$$

with equality if and only if $G=G_{0}$.

Led by those results, we believe that the graph $G_{0}$ has maximal spread in a more general case, i. e. that the following hypothesis holds.

Hypothesis 1. The graph $G_{0}$ (Fig. 6) is a unique graph with maximal spread in the class of connected graphs of order $n$ and size $n+k$ for $n>n_{0}$, where $n_{0}$ is a function on $n$ and $k$ $\left(n_{0} \in N, k \geq 0\right)$.

\section{References}

[1] T. M. Aleksić, M. Petrović, Cacti Whose Spread is Maximal, Graphs and Combinatorics, (2013) DOI 10.1007/s00373-013-1373-1.

[2] T. M. Aleksić, M. Petrović, Bicyclic Graphs Whose Spread is Maximal, Submitted to a journal.

[3] Y. Z. FAN, Y. WANG, Y. B. GAO, Minimizing the least eigenvalues of unicyclic graphs with application to spectral spread, Linear Algebra Appl. 429 (2008), 577-588.

[4] D. A. Gregory, D. Hershrowith, S. J. Kirkland, The spread of the spectrum of a graph, Linear Algebra Appl. 332“-334 (2001) 23-35.

[5] M. Petrović, On graphs whose spectral spread does not exceed 4, Publ. Inst. Math. 34 (48) (1983) 169-174.

[6] M. Petrović, B. BorovićAnin, T. Aleksić, Bicyclic graphs for which the least eigenvalue is minimal, Linear Alg. Appl. 430 (2009), 1328-1335.

[7] M. PEtrović, T. AleKsić, S. Simić, Further results on the least eigenvalue of connected graphs, Linear Algebra Appl. 435 (2011), 2303-2313. 